20xx IEEE. Personal use of this material is permitted. Permission from IEEE must be obtained for all other users, including reprinting/ republishing this material for advertising or promotional purposes, creating new collective works for resale or redistribution to servers or lists, or reuse of any copyrighted components of this work in other works 


\title{
Computationally-Efficient Iterative Demodulation of Coded PSK Signals Affected by Phase Noise
}

\author{
Nele Noels, Jabran Bhatti, Herwig Bruneel, Marc Moeneclaey \\ TELIN, Ghent University, Sint-Pietersnieuwstraat 41, 9000 Gent, Belgium \\ $\{$ nnoels, jb, hb, mm\} @telin. UGent.be
}

\begin{abstract}
This paper considers two recently-proposed receivers, Tikh and DCT. Both receivers are computationallyefficient, iterative and designed to be robust against phase noise on the local oscillators of digital bandpass communication systems. The presented results build on our prior research. We discuss the initialization of the DCT receiver, explore reducing the computational complexity by simplifying the receiver scheduling and study the effect of a small frequency offset. Coded PSK signaling and additive white Gaussian noise are assumed.
\end{abstract}

\section{INTRODUCTION}

In an ideal bandpass digital communication system, the local oscillators required for up-conversion at the transmitter and down-conversion at the receiver operate at exactly the same carrier frequency. In practice, however, there always is a mismatch between the carrier frequencies of the received signal and the sinusoid generated by the receiver oscillator; this mismatch is called the carrier frequency offset (FO). Moreover, non-ideal effects in the transmitter and receiver oscillators cause the carrier phase of the received baseband signal to show random fluctuations in time; this impairment is commonly known as phase noise (PN). As a result of FO and $\mathrm{PN}$, the received symbols are affected by a time-varying rotation, which, when not accounted for, yields an increased error probability.

Over the last decade, several iterative receiver algorithms have been developed for bit interleaved coded symbols transmitted over an additive white Gaussian noise (AWGN) channel affected by PN [1]-[12]. Among these algorithms, the Tikh receiver from [1] shows excellent performance at moderate complexity. In our prior work [12], we have proposed an alternative receiver that makes use of the discrete cosine transform (DCT) for compactly representing the time-varying phasor. As opposed to the receivers from [1], [2], [4], [6], [8], this DCT receiver does not involve recursive calculations, which makes it better suited for a parallel implementation on multiple cores.

The present paper extends the performance comparison of DCT and Tikh conducted in [12], with new results focussing on the initialization of the DCT receiver, the number of effective soft-input soft-output (SISO) demodulation steps and the robustness to a nonzero FO.

The paper is organized as follows. First, Section II describes the observation model. Then, Section III summarizes the DCT and Tikh receiver operations, after which Section IV elaborates on the receiver scheduling. Numerical results are shown in Section V and conclusions are drawn in Section VI.

Throughout this paper we use the following notations and conventions. The squared Euclidean norm of a vector of complex numbers is indicated as $|.|^{2}$ and $\mathbb{E}[\cdot]$ is the mean of a random variable. The transpose of a vector or matrix is referred to as $[\cdot]^{T}$. The operators $\cdot^{*}, \Re\{\cdot\}, \Im\{\cdot\}$ and $\arg \{\cdot\}$ represent the conjugate, real part, imaginary part and angle of a complex number. $\jmath$ denotes $\sqrt{-1}$ and $\mathbf{y}_{h ; H}$ is a short-hand notation for a vector $\left(y_{h, 0}, y_{h, 1}, \ldots, y_{h, H-1}\right)^{T}$ of dimension $H$, with $y_{h, l}=y_{(h-1) H+l}$, for $l=0,1, \ldots, H-1$.

\section{OBSERVATION MODEL}

We consider the transmission of a frame of $K$ PSK symbols over a bandpass channel affected by AWGN. At the receiver, a vector $\mathbf{r}_{1 ; K}=\mathbf{r}$ of $K$ matched filter output samples taken at the symbol rate is observed. We have, for $k=0, \ldots, K-1$,

$$
r_{k}=a_{k} u_{k}+n_{k}
$$

where $\mathbf{a}=\mathbf{a}_{1 ; K}$ is the transmitted symbol sequence, $\mathbf{u}=$ $\mathbf{u}_{1 ; K}$ collects the phasor samples and the vector $\mathbf{n}=\mathbf{n}_{1: K}$ consists of $K$ independent and identically distributed zeromean circular symmetric complex-valued Gaussian random variables with $\mathbb{E}\left[\left|n_{k}\right|^{2}\right]=N_{0}$. The carrier phasor samples are modeled as $u_{k}=e^{\jmath \theta_{k}}$, where $\left\{\theta_{k}\right\}$ is typically a discrete-time lowpass process [13] that stands for the instantaneous carrier phase shift of the received signal vis-a-vis the receiver's local reference carrier. The transmitted symbols belong to the $M$ PSK constellation $\Omega=\left\{\omega_{0}, \omega_{1}, \ldots, \omega_{M-1}\right\}$, with $\left|\omega_{i}\right|^{2}=E_{s}$, for $i=0,1, \ldots, M-1$, where $E_{s}$ denotes the symbol energy. To aid synchronization, $K_{p}$ known symbols (pilots) have been inserted in a at positions $k \in I_{p}=\left\{k_{j}: j=0,1, \ldots, K_{p}-1\right\}$, with

$$
k_{j}=\left\lfloor\frac{K(2 j+1)-K_{p}}{2 K_{p}}\right\rceil,
$$

where $\lfloor x\rceil$ rounds $x$ to the nearest integer ${ }^{1}$. The remaining $K-K_{p}$ symbols $a_{k}$ at positions $k \notin I_{p}$ represent unknown symbols (data), resulting from encoding an information bit sequence $\mathbf{b}$ of size $K_{b}$ and mapping the resulting coded bit sequence to a sequence of constellation points.

${ }^{1} x$ values with a fractional part of 0.5 round up to the nearest integer larger than $x$. 


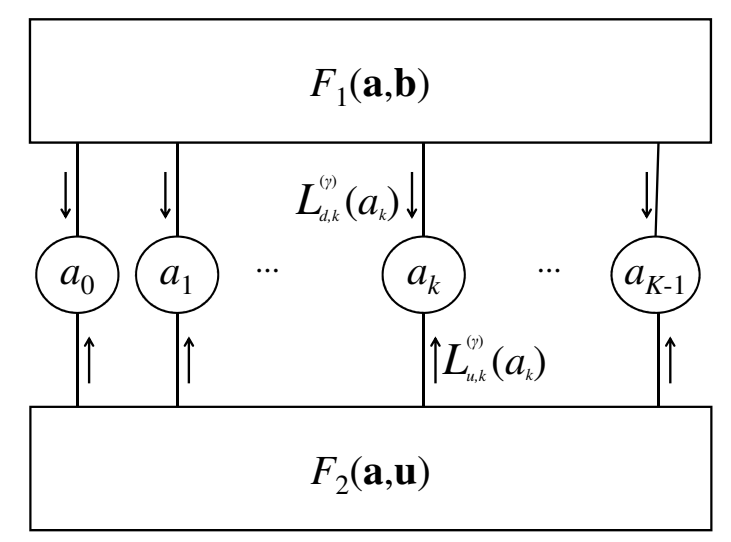

Figure 1. Graphical representation of iterative receiver for bit interleaved coded modulated signals.

\section{RECEIVER OPERATIONS}

\section{A. Main Factor Graph}

In the following, we assume that the reader is familiar with the factorgraph (FG)/sum-product algorithm (SPA) framework, see, e.g., [14]. Assuming independent equiprobable information bits, and dropping all factors not depending on $(\mathbf{b}, \mathbf{a}, \mathbf{u})$, the joint a posteriori probability (APP) $p(\mathbf{b}, \mathbf{a}, \mathbf{u} \mid \mathbf{r})$ of $\mathbf{b}, \mathbf{a}$ and $\mathbf{u}$, conditioned on $\mathbf{r}$ from (1) factorizes as:

$$
p(\mathbf{b}, \mathbf{a}, \mathbf{u} \mid \mathbf{r}) \propto F_{1}(\mathbf{a}, \mathbf{b}) F_{2}(\mathbf{a}, \mathbf{u}),
$$

where $F_{1}(\mathbf{a}, \mathbf{b})$ equals 1 when the code and mapping constraints map $\mathbf{b}$ to $\mathbf{a}$ and equals 0 otherwise, and $F_{2}(\mathbf{a}, \mathbf{u})=$ $\prod_{k} p\left(r_{k} \mid a_{k}, u_{k}\right) p(\mathbf{u})$ with $p\left(r_{k} \mid a_{k}, u_{k}\right)$ is the likelihood function of $\left(a_{k}, u_{k}\right)$, based on $r_{k}$, and $p(\mathbf{u})$ is the a priori distribution of $\mathbf{u}$. The corresponding FG is shown in Fig. 1 and forms the starting point for the development of many practical iterative receiver algorithms ${ }^{2}$. The messages from the factor nodes $(\mathrm{FN}) F_{1}(\mathbf{a}, \mathbf{b})$ and $F_{2}(\mathbf{a}, \mathbf{u})$ to a variable node $(\mathrm{VN})$ $a_{k}$ are denoted $L_{d, k}^{(\gamma)}\left(\omega_{i}\right)$ and $L_{u, k}^{(\gamma)}\left(\omega_{i}\right)$, respectively, where $i$ $=0,1, \ldots, M-1$ and $\gamma$ indicates the SPA iteration number ${ }^{3}$. By convention, these FG messages are assumed to be in the "logarithmic domain".

The downward messages $L_{d, k}^{(\gamma)}\left(\omega_{i}\right)$ result from applying the SPA to the FN $F_{1}(\mathbf{a}, \mathbf{b})$ using the upward messages $L_{u, k}^{(\gamma)}\left(\omega_{i}\right)$ as inputs. The former are in fact obtained as a by-product of the conventional joint SISO demapping and decoding operations. The SPA applied to the FN $F_{2}(\mathbf{a}, \mathbf{u})$ can be interpreted as a SISO demodulation process and involves the computation of the upward messages $L_{u, k}^{(\gamma)}\left(\omega_{i}\right)$ from the downward messages $L_{d, k}^{(\gamma-1)}\left(\omega_{i}\right)$. Two specific demodulation algorithms, referred to as Tikh and DCT, are considered next.

${ }^{2}$ For example, the algorithms from [1]-[6], [8], [9]. A different approach was taken in [10], [11]; these algorithms were specifically designed for trellisbased detectors and can therefore not be used, for example, for LDPC coded signals.

${ }^{3}$ Unless the FG is a tree, the SPA is iterative.

\section{B. Tikh}

In [1], the procedure for updating of $L_{u, k}^{(\gamma)}\left(\omega_{i}\right)$ results from assuming that, within an irrelevant constant, $p(\mathbf{u})$ further factorizes as $p(\mathbf{u})=\prod_{k} \exp \left\{-\frac{\left|u_{k}-u_{k-1}\right|^{2}}{2 \sigma_{P N, R x}^{2}}\right\}$. The parameter $\sigma_{P N, R x}$ is a design parameter, descriptive of the intensity of the Wiener PN process assumed by the receiver (see also Section V). Moreover, to reduce the computational complexity associated with applying the SPA to the resulting decomposition of FN $F_{2}(\mathbf{a}, \mathbf{u})$, some of the FG messages are approximated by Tikhonov distributions. Finally, the approximation $\log \left(I_{0}(|x|)\right) \approx|x|$ is used, where $I_{0}(\cdot)$ is the modified Bessel function of the first kind of the 0th order.

Tikh computes the messages $L_{u, k}^{(\gamma)}\left(\omega_{i}\right)$ as:

$$
L_{u, k}^{(\gamma)}\left(\omega_{i}\right)=\left|a_{f, k}+a_{b, k}+\dot{r}_{k}\left(\omega_{i}\right)\right| .
$$

Here,

$$
\begin{gathered}
\dot{r}_{k}\left(\omega_{i}\right)=\frac{2}{N_{0}} r_{k} \omega_{i}^{*} \\
a_{f, k}=\left(a_{f, k-1}+\xi_{k-1}\right)\left(1+\sigma_{P N, R x}^{2}\left|a_{f, k-1}+\xi_{k-1}\right|\right)^{-1} \\
a_{b, k}=\left(a_{b, k+1}+\xi_{k+1}\right)\left(1+\sigma_{P N, R x}^{2}\left|a_{b, k+1}+\xi_{k+1}\right|\right)^{-1}
\end{gathered}
$$$$
\text { with } \xi_{k}=r_{k} \alpha_{k}^{*}\left(N_{0}+E_{s}-\left|\alpha_{k}\right|^{2}\right)^{-1} \text {, where }
$$$$
\alpha_{k}= \begin{cases}\sum_{i=0}^{M-1} P_{d}\left(a_{k}=\omega_{i}\right) \omega_{i} & , k \notin I_{p} \\ a_{k} & , k \in I_{p}\end{cases}
$$

with $P_{d}\left(a_{k}=\omega_{i}\right)=e^{L_{d, k}^{(\gamma)}\left(\omega_{i}\right)} / \sum_{m=0}^{M-1} e^{L_{d, k}^{(\gamma)}\left(\omega_{m}\right)}$.

A block-processing implementation of Tikh is useful when a receiver is equipped with multiple cores that operate in parallel. Such an implementation requires a block-wise computation of the messages $L_{u, k}^{(\gamma)}\left(\omega_{i}\right)$, which implies that independent recursions are run to compute the parameters $a_{f, k}$ and $a_{b, k}$ over each block [4]. Because the values of these parameters are unknown at the block boundaries, all these recursions are initialized with zeros which in each block gives rise to a transient during which the accuracy of $a_{f, k}$ and $a_{b, k}$ is poor. A practical work-around, which reduces the performance degradation due to the transient effect at the expense of an increase in complexity, is the following. When $S$ cores are available, the receiver for frame size $K$ runs the recursions for computing $a_{f, k}$ and $a_{b, k}$ over subframes of size $L+2 W$ with $L=\frac{K}{S}$ and $W>0$. The $n$th subframe comprises the $n$th (non-overlapping) block of $L$ VNs $(k=n L, n L+1, \ldots,(n+1) L-1)$, the last $W$ VNs of the previous block (except when $n>0$ ) and the $W$ first VNs of the next block (except when $n<S$ ). The recursions for $a_{f, k}$ and $a_{b, k}$ are run over the entire subframe, yet the $W$ first and $W$ last values of $a_{f, k}$ and $a_{b, k}$ are discarded, and the messages $L_{u}^{(\gamma)}\left(\omega_{i}\right)$ are only computed for the $L$ remaining values of $k$.

${ }^{4}$ For completeness, we mention that, in contrast to Tikh, other FG-based receivers from [3] can be implemented in parallel without modification of the original algorithm; however, these receivers have been shown to be significantly more complex than the Tikh receiver. 


\section{C. $D C T$}

In [12], the procedure for updating of $L_{u, k}^{(\gamma)}\left(\omega_{i}\right)$ results from partitioning the received data frame into $S$ blocks of size $L$ (with $K=L S$ ) and assuming that the corresponding phasor subvectors can be represented by a linear combination of the first $N$ basis functions of the DCT, with typically $N \leq L$. Using matrix notation, this yields $\mathbf{u}_{h ; L}=\boldsymbol{\Psi}_{L \times N} \mathbf{x}_{h ; N}$, where $\boldsymbol{\Psi}_{L \times N}$ is an $L \times N$ DCT matrix and $\mathbf{x}_{h ; N}$ contains the $N$ coefficients of the truncated DCT expansion. Note that $\mathbf{x}_{h ; N}$ contains $L / N$ times less elements than $\mathbf{u}_{h ; L}$. The expectation-maximization algorithm (EMA) is subsequently used to produce estimates $\hat{\mathbf{x}}_{h ; N}$ of $\mathbf{x}_{h ; N}$ and the SPA is applied assuming $\hat{\mathbf{u}}_{h ; L}=\boldsymbol{\Psi}_{L \times N} \hat{\mathbf{x}}_{h ; N}$ is the true phasor, in which case the FN $F_{2}(\mathbf{a}, \mathbf{u})$ is replaced by $F_{2}(\mathbf{a}, \hat{\mathbf{u}})$, which further decomposes as $F_{2}(\mathbf{a}, \hat{\mathbf{u}}) \approx \prod_{k} \exp \left(\frac{2}{N_{0}} \Re\left\{r_{k} a_{k}^{*} \hat{u}_{k}^{*}\right\}\right)$, where the approximation holds for $\left|\hat{u}_{k}\right|^{2} \approx 1$. The EMA is an iterative procedure. Considering that also the SPA is iterative, we obtain a double-iterative receiver. In order to limit the receiver complexity, both types of iterations are intertwined.

At the $\gamma$ th iteration, DCT computes the messages $L_{u, k}^{(\gamma)}\left(\omega_{i}\right)$

$$
L_{u, k}^{(\gamma)}\left(\omega_{i}\right)=\Re\left\{\dot{r}_{k}\left(\omega_{i}\right) \hat{u}_{k}^{(\gamma-1) *}\right\}
$$

with

$$
\begin{gathered}
\hat{\mathbf{u}}_{h ; L}^{(\gamma)}=\boldsymbol{\Psi}_{L \times N} \boldsymbol{\Psi}_{L \times N}^{T} \mathbf{v}_{h ; L}^{(\gamma)}, \\
v_{k}^{(\gamma)}=\left\{\begin{array}{ll}
\frac{N_{0}}{2 E_{s}} \sum_{i=0}^{M-1} \dot{r}_{k}\left(\omega_{i}\right) p_{k}^{(\gamma)}\left(\omega_{i}\right), & k \notin I_{p} \\
\frac{N_{0}}{2 E_{s}} \dot{r}_{k}\left(a_{k}\right), & k \in I_{p}
\end{array},\right.
\end{gathered}
$$

and $p_{k}^{(\gamma)}\left(\omega_{i}\right)=e^{L_{d, k}^{(\gamma)}\left(\omega_{i}\right)+L_{u, k}^{(\gamma)}\left(\omega_{i}\right)} / \sum_{i=0}^{M-1} e^{L_{d, k}^{(\gamma)}\left(\omega_{i}\right)+L_{u, k}^{(\gamma)}\left(\omega_{i}\right)}$. In (8) and (10), $\dot{r}_{k}\left(\omega_{i}\right)$ is defined as in (5).

The parameters $L$ and $N$ are design parameters. In [12], it was shown that the performance of the DCT receiver is determined mainly by the ratio $N / L$ rather than by $N$ and $L$ separately.

DCT is well-suited to exploit the presence of multiple cores. Because all operations are performed independently on different blocks, they can be split over different cores without need for these cores to act in synchrony or to exchange information. When $S$ cores are available, for given frame size $K$ the receiver selects the block size $L$ as $L=K / S$ so that each core processes a single block per frame.

\section{RECEIVER SCHEDULING}

In order to start the DCT iterations, an initial phasor estimate $\hat{\mathbf{u}}^{(0)}$ is required. In [12], this estimate is obtained from the observations $r_{k}, k \in I_{p}$ at the pilot symbol positions only. Such an estimate is referred to as pilot-aided (PA) and denoted $\hat{\mathbf{u}}^{\langle P A\rangle}$. As far as the initial estimate is concerned, the received signal vector $\mathbf{r}$ is divided into $S_{p}$ blocks of length $L_{p}$ with $S_{p} L_{p}=K$; note that $L_{p}$ might be different from the block length $L$ used during the iterations. Each block contains $B_{p}$

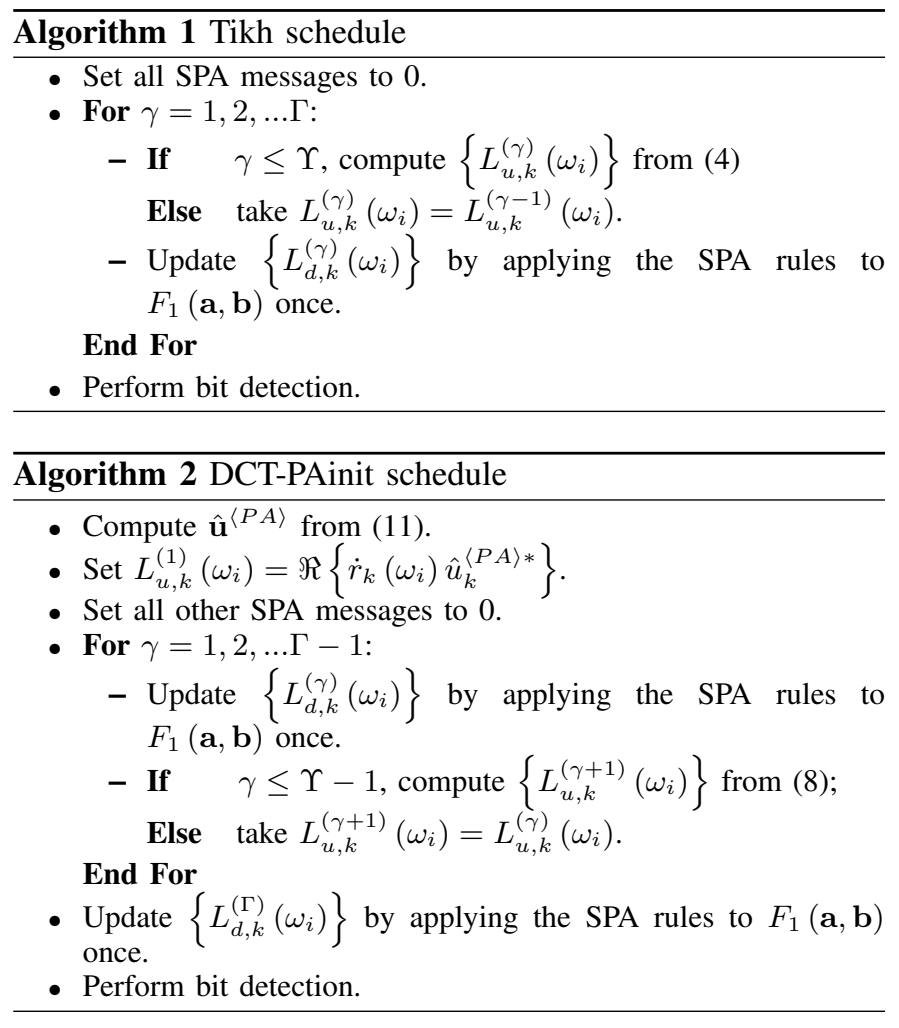

pilot symbols such that $S_{p} B_{p}=K_{p}$. The PA phasor estimate is determined by

$$
\hat{\mathbf{u}}_{h ; L_{p}}^{\langle P A\rangle} \approx\left(K / K_{p}\right) \boldsymbol{\Psi}_{L_{p} \times N_{p}} \boldsymbol{\Psi}_{p}^{T}\left(\mathbf{r}_{h ; L_{p}} \circ \mathbf{a}_{h ; L_{p}}^{*}\right),
$$

where $\circ$ denotes element-wise multiplication, $\boldsymbol{\Psi}_{L_{p} \times N_{p}}$ is the $L_{p} \times N_{p}$ DCT matrix, and $\boldsymbol{\Psi}_{p}$ is an $L_{p} \times N_{p}$ matrix obtained by replacing in $\Psi_{L_{p} \times N_{p}}$ the $\left(L_{p}-B_{p}\right)$ rows that correspond to data symbol positions with zero vectors. The approximation (11) holds for the considered pilot symbol positions (2). In [12], it was shown that the mean square estimation error resulting from (11) can be minimized by properly selecting the ratio $N_{p} / B_{p}$.

An alternative way to compute an initial estimate $\hat{\mathbf{u}}^{(0)}$ is by running (9) for $\gamma=0$ with $L_{d, k}^{(0)}\left(\omega_{i}\right) \equiv 0$ and $L_{u, k}^{(0)}\left(\omega_{i}\right)$ given by (8) with $\gamma=0$ and $\hat{\mathbf{u}}^{(-1)}=\hat{\mathbf{u}}^{\langle P A\rangle}$ from (11). The resulting initial estimate is based on all observations rather than on the observations at pilot symbol positions only, meaning that $\hat{\mathbf{u}}^{(0)}$ is potentially more accurate than $\hat{\mathbf{u}}^{\langle P A\rangle}$. Because no a priori information on the coding stucture is exploited in the estimation process, we refer to this type of initialization as non-code-aided (NCA).

The receiver scheduling of Tikh, DCT with PA initialization (DCT-PAinit) and DCT with NCA initialization (DCTNCAinit) is summarized in Algorithms 1-3. Applying the SPA updating rules to $F_{1}(\mathbf{a}, \mathbf{b})$ includes computing information bit a posteriori probabilities that can be subsequently used to perform maximum a posteriori bit detection.

As a further simplification of the receivers, we also consider the possibility to update the upward messages $\left\{L_{u, k}^{(\gamma)}\left(\omega_{i}\right)\right\}$ 


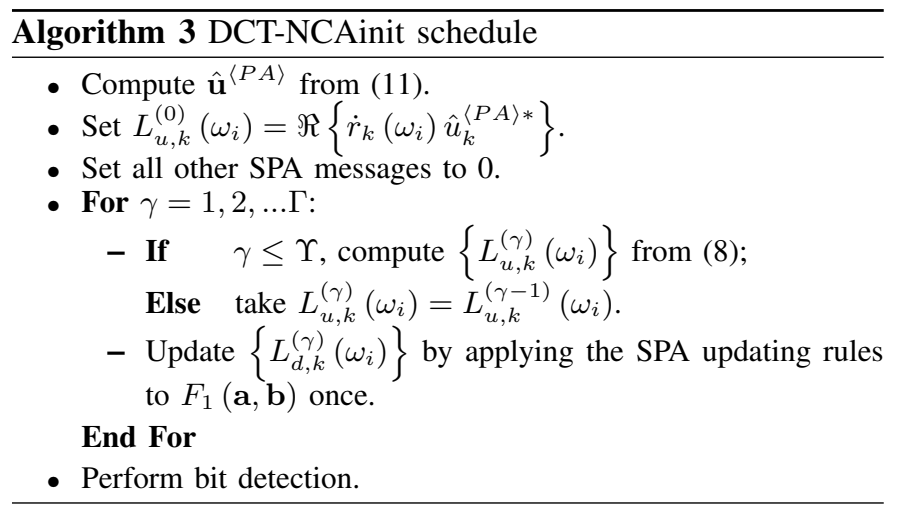

during only the first $\Upsilon$ (Tikh and DCT-NCAinit) or $\Upsilon-1$ (DCT-PAinit) iterations, and freeze these messages during the last $\Gamma-\Upsilon$ iterations; the downward messages $\left\{L_{d, k}^{(\gamma)}\left(\omega_{i}\right)\right\}$ are updated during all iterations ( $\Gamma$ times). Note that, for given $\Upsilon$, DCT-NCAinit requires $(\Upsilon+1)$ evaluations of the messages $\left\{L_{u, k}^{(\gamma)}\left(\omega_{i}\right)\right\}$ while for Tikh and DCT-PAinit this is only $\Upsilon$.

\section{Numerical Results AND Discussion}

We now present numerical results comparing the error performance of Tikh, DCT-PAinit and DCT-NCAinit, for the case where the actual phase sample trajectory contains a contribution caused by a non-zero FO, while the design parameters $\left(N / L\right.$ or $\left.\sigma_{P N, R x}\right)$ were optimized under the assumption that $F=0$. These results complement our previous work [12]. The error performance will be measured in terms of the bit error rate (BER) and the signal-to-noise ratio is expressed as $E_{b} / N_{0}$, with $E_{b}=K E_{s} / K_{b}$ the energy per information bit. Pseudo-random bit interleaved coded 8PSK modulation is considered. The carrier phase samples are modelled as $\theta_{k}=\theta_{k-1}+2 \pi F T+\phi_{k}$, with $\theta_{0}$ uniformly distributed in $\left[-\pi, \pi\left[,\left\{\phi_{k}\right\}\right.\right.$ statistically independent Gaussian random variables with zero mean and standard deviation $\sigma_{P N}, F$ a FO and $T$ the symbol period.

We first consider a rate-2/3 irregular repeat accumulate LDPC code with $K_{b}=43200$, specified in the DVB-S2 standard [15], and take $K_{p}=540$, yielding $K=22140$. Fourthy receiver iterations are performed and the messages $\left\{L_{u, k}^{(\gamma)}\left(\omega_{i}\right)\right\}$ are updated at each iteration, i.e., $\Upsilon=\Gamma=40$. In [12], we found that, when the receiver is equipped with $S=K / L$ cores and each core processes a single block per frame, Tikh requires a longer parallel execution time than DCT-PAinit when both receivers use a high degree of parallelization $(45 \leq L \leq 246)$.

Fig. 2 shows the BER of DCT-PAinit with $N=2$ and Tikh with $W \in\{L, L+20\}$ as a function of $E_{b} / N_{0}$, for $L=54$, $\sigma_{P N}=3^{\circ}$ and $F T \in\left\{0,1.25 \cdot 10^{-3}\right\}$. We observe that the faster DCT-PAinit receiver outperforms the slower Tikh receiver when $W=0$. Increasing $W$ to $W=10$ yields a power efficiency gain of $0.45 \mathrm{~dB}$ for Tikh, but also an increase in parallel computation time of almost $50 \%$ (see also [12]). Increasing the FO from 0 to $1.25 \cdot 10^{-3} / T$ degrades the BER performance of both Tikh and DCT-PAinit. However,

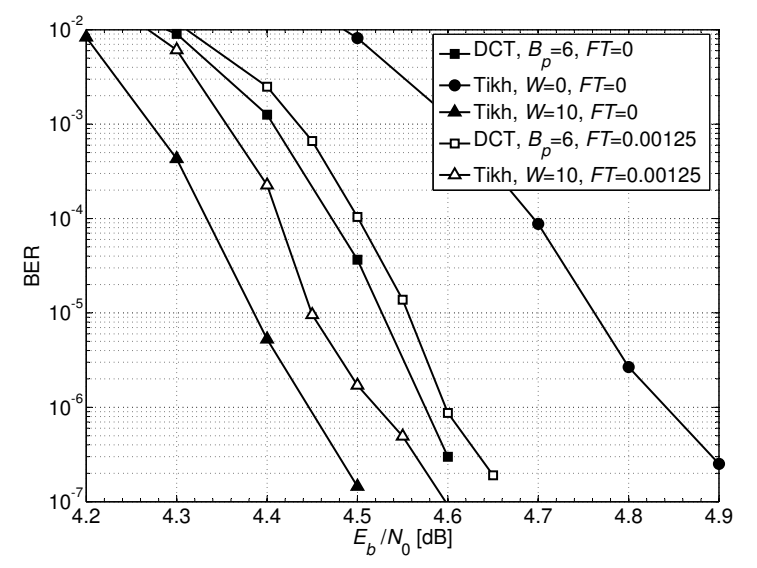

Figure 2. BER performance of Tikh with $W \geq 0$ and of DCT-PAinit with $N=N_{p}=2$ as a function of $E_{b} / N_{0}$, for several values of $F$ and for $L=54, \Gamma=\Upsilon=40, \sigma_{P N}=3^{\circ}$ and LDPC coded 8PSK signalling.

this degradation is more significant for Tikh with $W=10$ than for DCT-PAinit, which implies that the advantage of Tikh with $W=10$ over DCT-PAinit in terms of BER performance decreases with increasing $F T$.

Above we have compared DCT and Tikh in a parallel implementation setting, where the receiver is equipped with multiple cores and each core processes a single block per frame. However, in conventional single-core receivers, all blocks are processed sequentially rather than in parallel. In such a case, there is no reason for using Tikh with $L<K$, which causes only performance degradation and no complexity reduction as compared to Tikh with $L=K$. It was illustrated in [12] that, in such a case, DCT with small $L$ may still provide an interesting low-complexity alternative to Tikh with $L=K$. The considered system was a rate $1 / 3$ turbo code with $K_{b}=1000$ information bits that consists of the parallel concatenation of two identical rate $1 / 2$ recursive systematic convolutional codes with generator sequences (11111) and (10001). In this case, a multi-core receiver implementation is cost-inefficient because the trellis-based turbo decoding algorithm itself involves recursive calculations that prevent parallel computation. In [12], a fixed amount of $\Gamma=10$ SPA iterations was carried out and at each iteration the upward messages $\left\{L_{u, k}^{(\gamma)}\left(\omega_{i}\right)\right\}$ were updated. $K_{p}=50$ pilot symbols were inserted, $\sigma_{P N}$ was assumed to be $2^{\circ}$ and no FO was considered $(F=0)$. For Tikh, $L$ was set equal to $K$ and the parameter $\sigma_{P N, R x}$ was choosen equal to $\sigma_{P N}$. For DCT, $N_{p}$ and $N$ were both set to 2 and $\left(L_{p}, L\right) \approx(360,100)^{5}$ was selected to minimize the BER at $E_{b} / N_{0}=3 \mathrm{~dB}$. Under these circumstances it was concluded that the computational complexity of the SISO demodulation process (per symbol and per iteration) is significantly higher for Tikh with $L=K$ than for DCT with $L=100$ and $N=2$ (131 versus 86 operations

${ }^{5}$ Because 360 is not an integer divisor of 1000 , for DCT initialization we split the frame into two blocks of size 360 and one block of size 280 . 


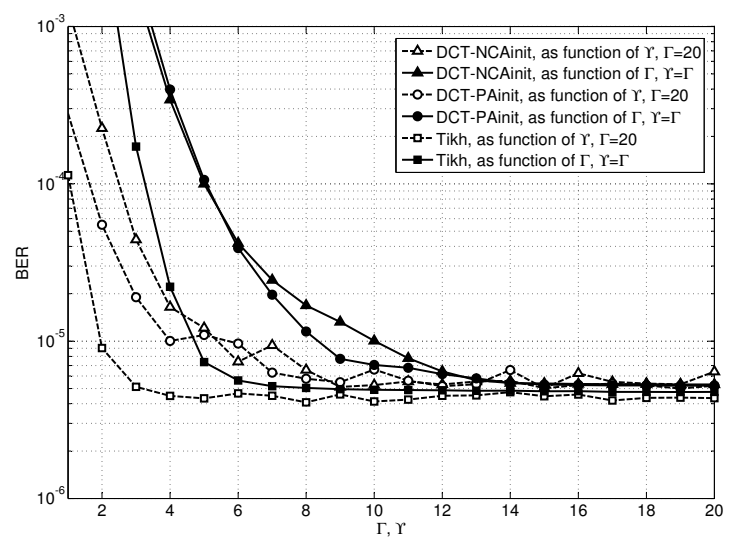

Figure 3. BER of Tikh with $L=K$ and of DCT with $N=N_{p}=2$ and $\left(L, L_{p}\right)=(100,360)$ at $E_{b} / N_{0}=3 \mathrm{~dB}$, for $\sigma_{P N}=2^{\circ}, F=0$ and turbo coded 8PSK signalling, as a function of the number of iterations.

per symbol and per iteration, i.e., about 50\% difference).

The results presented next put these conclusions into perspective. Fig. 3 shows the corresponding BER for Tikh with $L=K$ and for DCT-PAinit and DCT-NCAinit with $L=100$ and $N=2$ at $E_{b} / N_{0}=3 \mathrm{~dB}$ as a function of $\Gamma$ for $\Upsilon=\Gamma$ and as a function of $\Upsilon$ for $\Gamma=20$. We observe that

- For $\Upsilon=\Gamma$, Tikh requires significantly less (only half as much) decoding iterations than DCT to converge to a steady state BER value.

- For $\Gamma=20$, Tikh requires significantly less (only half as much) updates of the messages $\left\{L_{u, k}^{(\gamma)}\left(\omega_{i}\right)\right\}$ than DCT.

- DCT-NCAinit does not converge faster nor to a lower steady-state BER value than the less complex DCTPAinit.

As far as the DCT receivers are concerned it should be noted that the first evaluation of the upward messages involves operations that are performed on the pilot symbols only. The contribution of these operations to the overall complexity is negligible in the usual case where the ratio $K / K_{p}$ is large (here, 20). From the above we can conclude that, at least for this particular set-up, using DCT instead of Tikh yields hardly any net complexity gain.

\section{CONCLUSIONS AND REMARKS}

The Tikh receiver from [1] and the DCT receiver from [12] perform computationally-efficient iterative decoding in the presence of phase noise. Both receivers iterate between a SISO decoder (the same for both receivers) and a SISO demodulator (different for Tikh and DCT) that exchange soft information. In the absence of a frequency offset and assuming that the soft information at the output of the SISO demodulator is updated at each receiver iteration, it was shown in [12] that DCT with PA initialization can provide a computationally efficient alternative to Tikh. This paper further discusses the initialization, the scheduling and the robustness of these receivers. Results obtained for LDPC coded 8PSK with $2.5 \%$ pilot symbols and a parallel receiver implementation on 54 cores (each processing a different part of the received frame) show that a nonzero frequency offset causes a larger BER degradation for Tikh than for DCT. Results obtained for turbo coded 8PSK with 5\% pilot symbols and a conventional sequential receiver implementation further indicate that the complexity of both DCT and Tikh can be reduced by updating the SISO demodulator output during only the first $\Upsilon$ iterations, and freeze its value during the last $\Gamma-\Upsilon$ iterations, while the SISO decoder output is updated during all $\Gamma$ iterations. For the considered set-up and as compared to DCT, Tikh requires roughly half as many SISO demodulator output updates to converge to a steady state BER value. Finally, our results indicate that DCT with NCA initialization not necessarily outperforms the less complex DCT with PA initialization.

\section{ACKNOWLEDGMENT}

This research has been funded by the Interuniversity Attraction Poles Program initiated by the Belgian Science Policy Office. The first author would like to acknowledge the financial support of the Fund for Scientific Research - Flanders.

\section{REFERENCES}

[1] G. Colavolpe et al., "Algorithms for iterative decoding in the presence of strong phase noise," IEEE J. Sel. Areas Commun., vol. 23, no. 9, pp. 1748-1757, Sep. 2005.

[2] J. Dauwels and H.-A. Loeliger, "Phase estimation by message passing," in 2004 IEEE Int. Conf. Commun., Paris, France, 2004, pp. 523-527.

[3] G. Colavolpe, "On LDPC codes over channels with memory," IEEE Trans. Wireless Commun., vol. 5, pp. 1757-1766, Jul. 2006.

[4] A. Barbieri et al., "Joint iterative detection and decoding in the presence of phase noise and frequency offset," IEEE Trans. Commun., vol. 55, pp. 171-179, Jan. 2007.

[5] L. Benvenuti et al., "Code-aware carrier phase noise compensation on turbo-coded spectrally-efficient high-order modulations," in 8-th Int. Workshop on Signal Process. Space Commun., Catania, Italy, 2003, pp. 177-184.

[6] N. Noels et al., "Performance analysis of ML-based feedback carrier phase synchronizers for coded signals," IEEE Trans. Signal Process., vol. 55, pp. 1129-1136, Mar. 2007.

[7] R. Krishnan et al., "Soft metrics and their performance analysis for optimal data detection in the presence of strong oscillator phase noise," IEEE Trans. Commun., vol. 61, pp. 2385-2395, Jun. 2013.

[8] N. Kamiya and E. Sasaki, "Pilot-symbols assisted and code-aided phase error estimation for high-order QAM transmission," IEEE Trans. Commun., vol. 61, pp. 4369-7380, Oct. 2013.

[9] J. Bhatti et al., "Algorithms for iterative PN estimation based on a truncated DCT expansion," in IEEE 12th Int. Workshop Signal Process. Advances in Wireless Commun., San Francisco, CA, 2011, pp. 51-55.

[10] G. Ferrari et al., "On linear predictive detection for communications with phase noise and frequency offset," IEEE Trans. Veh. Technol., vol. 56, pp. 2073-2085, Jul. 2007.

[11] A. Anastasopoulos and K. M. Chugg, "Adaptive iterative detection for phase tracking in turbo coded systems," IEEE Trans. Commun., vol. 49, pp. 2135-2144, Dec. 2001.

[12] N. Noels et al., "Block-processing soft-input soft-output demodulator for coded psk using dct-based phase noise estimation," IEEE Trans. Commun., vol. 62, no. 8, pp. 2939-2950, Aug. 2014

[13] A. Chorti and M. Brookes, "A spectral model for RF oscillators with power-law phase noise," IEEE Trans. Circuits Syst. I: Reg. Papers, vol. 53, pp. 1989-1999, Sep. 2006.

[14] H.-A. Loeliger, "An introduction to factor graphs," IEEE Signal Processing Mag., vol. 21, no. 1, pp. 28-41, Jan. 2004

[15] Digital Video Broadcasting (DVB); Second generation framing structure, channel coding and modulation systems for Broadcasting, Interactive Services, News Gathering and other broadband satellite applications (DVB-S2), ETSI Std. EN 302307 v1.3.1, 2013. 